\title{
Active Mutation in Self-reproducing Networks of Machines and Tapes
}

\author{
Takashi Ikegami *and Takashi Hashimoto ${ }^{\dagger}$ \\ Institute of Physics, College of Arts and Sciences, \\ The University of Tokyo, \\ 3-8-1, Komaba, Meguro-ku, Tokyo 153, Japan \\ Tel \& Fax: +81-3-5454-6535
}

May 31, 1996

\begin{abstract}
A self-reproduction via description is discussed in a network model of machines and description tapes. Tapes consist of bit strings, encoding function of machines. A tape is replicated when it is read by an adequate machine. Generally, a machine rewrites a tape without doing correct replication. The variation in a reproduced tape is taken as mutation. Since this mutation is caused by a machine's program, we call it active mutation. Which machine is translated from a given tape is dependent on what kind of a machine reads the tape. External noise is introduced in machine's reading process to make errors. A new reaction pathway is induced by external noise via machine's error action. We find that the induced pathways will be mimicked deterministically in an emerging core structure. This core structure will be remained stable after turning off external noise. Low external noise develops a core structure of minimal self-replicative loop. When external noise is elevated, a more complex network evolves. Machines composing a complex core network, which has been bred in high external noise, will actively rewrite tapes rather just replicate them. Self-replication not as an individual but as a network now becomes important.
\end{abstract}

Key words: Active mutation; Self-reproduction; Machines; Tapes; Core network; Double loop replication

\footnotetext{
${ }^{*}$ E-mail address : ikeg@sacral.c.u-tokyo.ac.jp

${ }^{\dagger}$ E-mail address : toshiwo@sacral.c.u-tokyo.ac.jp
} 


\section{Introduction}

Reproduction and mutation are both sides of one thing. Mutation is not just brought by the external causes. It is resulting from the inevitable nature of self-reproduction. Self-reproduction at the same time induces mutation, which we call active mutation. In order to pursue this idea, we study a self-reproduction dynamics with description.

In order to replicate a complex active entity such as a living cell, to make the description is prerequisite. For example, living systems have elaborated their own description, DNA.

However description is not always easy to obtain. Some sort of "observation" process is needed to make description. But some objects are easily destroyed by observation itself. Difficulty of making description is not only attributed to the object itself. How we observe determines which objects can or cannot be allowed to have description.

Reproduction by itself provides far difficult problems. An object and an observer are no more separated entities. Self-reproductive objects should observe themselves to copy themselves. Such self-observation leads to endless regression. Namely, a machine observes itself, which observes itself, which observes $\cdots$.

One resolution has been shown by von Neumann[19] that a self-reproduction can be possible with a universal constructing machine appended with a description and a copier machine. A universal constructing machine copies itself and a copier by reading the description. The copier just copies the description without translation.

On the other hand, Chris Langton discusses that a naturally occurring self-reproduction may not use a universal constructor nor a description [17]. In order to distinguish a biological reproduction from a mere crystal growth, some non-triviality in a reproduction form is required. As an example, Langton has shown a self-reproduction of loop configurations in 2-dimensional Cellular Automata (CA). A separation of machines and description is not necessary for self-reproduction in that case. ${ }^{1}$

If a unique CA configuration is able to replicate as in Langton's system, we merely prepare a description of the unique reproducing "machine". We admit that in such a case a separation of machine and description does not matter at all. But if more than two possible reproducing machines interfere

\footnotetext{
${ }^{1}$ Recently, it is shown that extra information can be copied with the loop configuration by slightly modifying CA rules [18]. This makes a tape more close to what we call description.
} 
with each other, we have to care about the followings:

- Syntax mixture

A tape a becomes a description of a machine $\mathbf{A}$ when it is read by a machine A. But it can be translated into different machines or fails to reproduce when it is read by the different machines. How can machines find their own right description?

- Semantics mixture

If a tape $\mathbf{a}$ expresses "A tape $\mathbf{b}$ is wrong" and a tape $\mathbf{b}$ expresses " $A$ tape $\mathbf{a}$ is right", we face with a paradoxical situation. When tapes can carry information more than reproduction information, such semantics mixture causes serious paradoxes.

- Evolution and mutation

A separation of machines and tapes is relevant for evolution. If a machine can rewrite tapes to make new machines, evolution can be discussed as a deterministic read/write process. This is what we will see in this paper.

- Stability of reproduction and erroneous actions

A self-reproducing configuration in von Neumann's or Langton's CA is structurally unstable. A slight difference in the reproducing configuration fails to show any reproduction. Such fragilty of reproduction causes a serious problem for realistic situation. Further if machines can make mistakes when reading tapes, reproduction is easily terminated. To overcome such fragilty is an urgent issue.

In this paper, we will study the evolution of machines and description tapes influenced by random external noise $[1,2,3]$. While a machine reads a tape, both probabilistic and deterministic mutations are assumed to occur. The probabilistic mutation is caused by external noise and is called a passive mutation. On the other hand, the deterministic mutation is caused by machine action and is named active mutation.

In low external noise regime, perfect replicating network composed of one or two machine(s) is evolved. In high noise regime, a complex autocatalytic network sustaining deterministic mutation evolves. Self-replication not as an individual but as a network now becomes important. In the other autocatalytic models $[6,4]$, reproduction is discussed only by machines or 
by tapes. However, the important fact is that no machine can reproduce itself without the coding tapes and vice versa. Because of this restriction, autocatalytic loops only of machines or tapes are inhibited. By considering both machines and tapes, we can start discussing the dynamics of encoding and decoding process in reproduction.

\section{Modeling}

Our system consists of two different objects, tapes and machines.

A tape has a bit string of a circular form. A machine consists of 3 different parts, a head, a tail and a transition table. Each head and tail is expressed by a 4 bit string, whose pattern will be compared with binary patterns of tapes. A transition table consists of 4 mappings; $\left(\sigma^{m}, \sigma^{t}\right) \rightarrow\left(\sigma^{\prime m}, \sigma^{\prime t}\right)$, where $\sigma^{m}$ and $\sigma^{t}$ represent current binary state of machine and tape, respectively. A tape and machine state will change to $\left(\sigma^{\prime m}, \sigma^{\prime t}\right)$ depending on a current state of machine and tape $\left(\sigma^{m}, \sigma^{t}\right)$.

Introducing an ensemble of tapes and machines, we carry out a machinetape reaction process as follows:

(1) Interaction of machines and tapes:

A machine $\mathbf{M}_{i}$ reads a tape $\mathbf{T}_{j}$ iff the tape $\mathbf{T}_{j}$ has the same pattern of head $\mathbf{h}_{i}$ and tail $\mathbf{t}_{i}$ of the machine $\mathbf{M}_{i}$ in a different site of the tape $\mathbf{T}_{j}$. The sites from a first bit of $\mathbf{h}_{i}$ to that of $\mathbf{t}_{i}$ will be called the reading frame.

Then machine $\mathbf{M}_{i}$ rewrites the reading frame according to its own transition table. A half population of machine starts to read a tape with the internal state 1 and the other half does with the state 0 . We assume that there exist a sufficient amount of resources so that a pair of machine and tape can unlimitedly generate a new tape $\mathbf{T}_{l}$ and a machine $\mathbf{M}_{k}$ translated from that tape.

$$
\mathbf{M}_{i}+\mathbf{T}_{j} \Rightarrow \mathbf{M}_{k}+\mathbf{T}_{l}+\mathbf{M}_{i}+\mathbf{T}_{j}
$$

(2) Translation of tapes:

Not only bits of a reading frame, but every bit of tape is repeatedly picked up to construct a new machine from a first site of the reading frame. If a length of a tape is not enough, the same bit is used for coding several different part of a machine. In the present model, we use a fixed length of 7-bit tapes and 16-bit machines. A first 8 bits are mapped onto head and tail parts in order. The next 8 bits are mapped onto a transition table. In order to cover 16 bits by 7 bits, several bits are used twice. In Fig. 1, we 
give an example of how a machine rewrites a tape and how a new machine is translated from the rewritten tape.

Each tape has a source where an attached machine starts to search for the head and tail pattern. Starting from the site, patterns are searched for in the clockwise direction of a circular tape. When a head pattern is found, a tail pattern starts to be searched in the clockwise direction. The site of source can be updated randomly when the tape is newly generated after its extinction. An identical tape with different source can make different machines being read by the same machine. But here every coexisting translational invariant tape has the same site of a source.

(3) Population dynamics:

We assume a finite capacity $N$ for both tapes and machines. By iterating the following procedures, we simulate the machine/tape reactions:

1. Compute concentration of machines and tapes by dividing the population number of each object, denoted by $m_{i}, t_{i}$, respectively, by the capacity size $N$ as following equations,

$$
\begin{gathered}
f_{i}^{M}=\frac{m_{i}}{N}, \\
f_{j}^{T}=\frac{t_{j}}{N},
\end{gathered}
$$

where, $f_{i}^{M}$ and $f_{j}^{T}$ is concentration of $i$-th machine and $j$-th tape, respectively.

2. Make a total $c N$ numbers of new machines and tapes from reaction of machines and tapes. Here the coefficient $c$ gives a rate of new machines in a total capacity $N$. One generation is defined as the period needed to make $c N$ new machines and tapes. The rate of reaction $f_{i j}$ is given by,

$$
f_{i j}=\frac{c f_{i}^{M} f_{j}^{T}}{\sum_{k, l} f_{k}^{M} f_{l}^{T}} .
$$

3. Remove $d_{m} \%$ of old machines and $d_{t} \%$ of old tapes.

4. Put the new machines and tapes back in each space. Hence the population of machine $i$ and tape $j$ of the next generation becomes,

$$
m_{i}^{\prime}=\left(1-d_{m}\right) m_{i}+\sum_{k+j \rightarrow i} f_{k j} N
$$


(1)
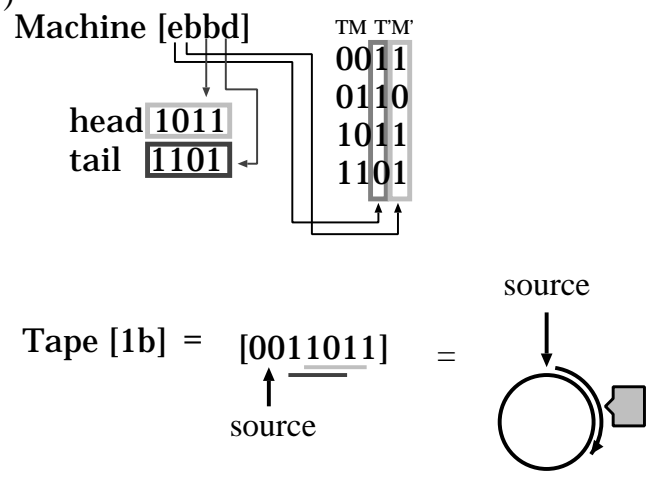

(2)

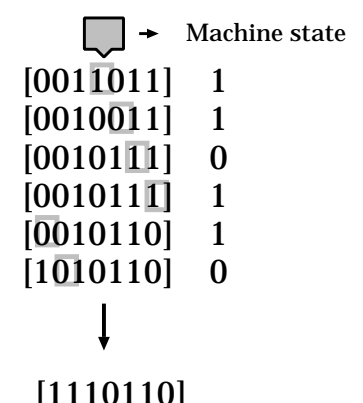

(3)

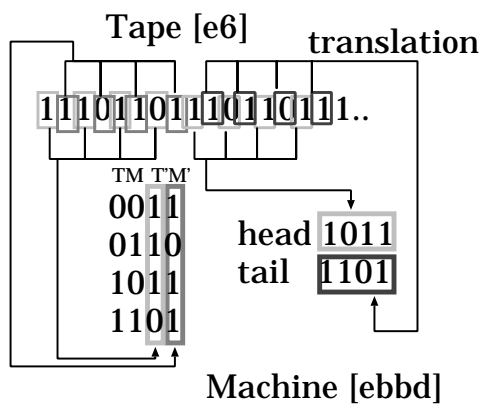

Figure 1: An example of a rewriting process. Here the machine $\mathbf{M}_{\mathbf{e b b d}}$ reads the tape $\mathbf{T}_{1 \mathbf{b}}$, and generating a new tape $\mathbf{T}_{\mathbf{e} 6}$ with a new machine $\mathrm{M}_{\text {ebbd }}$.

1)Structures of a machine $\mathbf{M}_{\text {ebbd }}$ and a tape $\mathbf{T}_{1 b}$ are depicted. A transition table determines a transition rule $\left(\sigma^{m}, \sigma^{t}\right) \rightarrow\left(\sigma^{\prime m}, \sigma^{\prime t}\right)$, where $\sigma^{m}$ and $\sigma^{t}$ represent current binary state of machine and tape, respectively. A head pattern [1011] and a tail pattern [1101] of a machine $\mathbf{M}_{\mathbf{e b b d}}$ are found on different sites of a tape $\mathbf{T}_{1 \mathbf{b}}$. Hence the tape is transcribed by the machine. 2) During the transcription process, several bits are rewritten due to the machine's transition table. Here the machine starts to read the tape with the initial state 1. A small box on each string denotes the machine's reading site. A machine always move in a clockwise direction.

3) The transcribed tape is translated from the first site of a reading frame. Each bit is succesively translated into machine's transition table, a head and a tail pattern. 


$$
t_{j}^{\prime}=\left(1-d_{t}\right) t_{j}+\sum_{k+i \rightarrow j} f_{k i} N
$$

If no reactions occur, the second terms in the above equations vanish. It should be noted here that each machine has its unique description tape but the inverse is not true. Generally a tape encodes several machines depending on which machine reads the tape.

5. Taking an integer part of the above population, we obtain the actual population of the next generation. Hence the machine or tape whose concentration $\left(f_{i}^{M}, f_{j}^{T}\right)$ is lower than $N^{-1}$ is removed from the population.

(4) Effect of external noise:

Out of $c N$ new tapes, a number of tapes as well as machines, are erroneously generated by external noise. It is assumed that the rate of mutation depends on the reading frame. Namely, the rate of mutation replication by external noise is given by,

$$
\varepsilon=1-\left(1-\mu_{P}\right)^{L},
$$

where the symbol $L$ is the length of reading frame. We use the Monte Carlo method to get the mutant objects. At most $\varepsilon c N$ mutant populations are generated by randomly flipping the bit within a reading frame. As the result, the reaction terms in the equations (7) and (8) are decreased by the factor of $1-\varepsilon$ and noise generated populations are added.

(5)Active mutation:

During the read/write process, both probabilistic and deterministic mutations are assumed to occur. The probabilistic mutation is caused by external noise and is called passive mutation. On the other hand, the deterministic mutation is caused by machine action, and is named active mutation. We call it mutation since it does not completely replicate a tape but actively rewrites it. A rewritten tape can be taken as a mis-copy of the original tape. The rate of passive mutation, denoted by $\mu_{P}$, is measured by a bit flip rate per a bit. The active mutation per a reaction is measured by the rewriting rate per length of reading frame when a machine $i$ reads a tape $j$. Namely, it is given by,

$$
\mu_{A i j}=\frac{w}{L_{i j}}
$$


where a symbol $w$ denotes the number of rewritten bits and $L_{i j}$ denotes length where machine $i$ reads a tape $j$. Averaged active mutation rate is given by,

$$
\left\langle\mu_{A}\right\rangle=\frac{\sum_{i j} \mu_{A i j} m_{i} t_{j}}{\sum_{i j} m_{i} t_{j}} .
$$

\section{Destabilization of a minimal self-replicating loop}

About 10 randomly selected machines with 2 or 3 tapes are prepared as an initial configuration. A machine without description tape is unstable and smoothly removed from the system. Hence an initial configuration which does not include any description tapes of the initial machines will die out. External noise may produce description tapes by mistake.

Even without external noise, a machine can acquire its tape by the other machines' product. To sustain the tape, we have to make the tape of the machine which generates the tape of the first machine. In order to complete it, a successive reproducing process should form a closed loop; each machine on the loop reproduces a machine for the next position.

We will see how the replicating networks evolve by changing the amount of external noise. Examples of temporal evolution of population of machines and tapes are shown in Fig. 2.

By introducing a lower amount of external noise in a system, we see a minimal autocatalytic loop evolve. In this example, a machine $\mathbf{M}_{1002}$ reads a tape $\mathbf{T}_{1}$ to replicate the tape $\mathbf{T}_{1}$ and the machine $\mathbf{M}_{\mathbf{1 0 0 2}}$. The number attached to tapes and machines are hexadecimal number converted from its binary representation. Many initial configurations reach this minimal autocatalytic system for a lower noise regime.

A system with the minimal autocatalytic loop is said to be metastable since it remains stable after turning off external noise but destabilized by increasing external noise. Reaction under external noise generates many machines, most of them are parasitic machines. Namely, machines cannot replicate without being supported by other machines. Increasing parasite machines weakens the original self-replicating loop. In Fig. 2-a), a parasitic machine $\mathbf{M}_{1222}$ invades the network. Its description tape $\mathbf{T}_{\mathbf{3}}$ is also increasing its population.

A greater variety of machines and tapes induces unstable oscillation in Fig. 2-b). An original self-replicating pair becomes unstable if too many parasitic machines attach to it. Population of each machine and tape show 

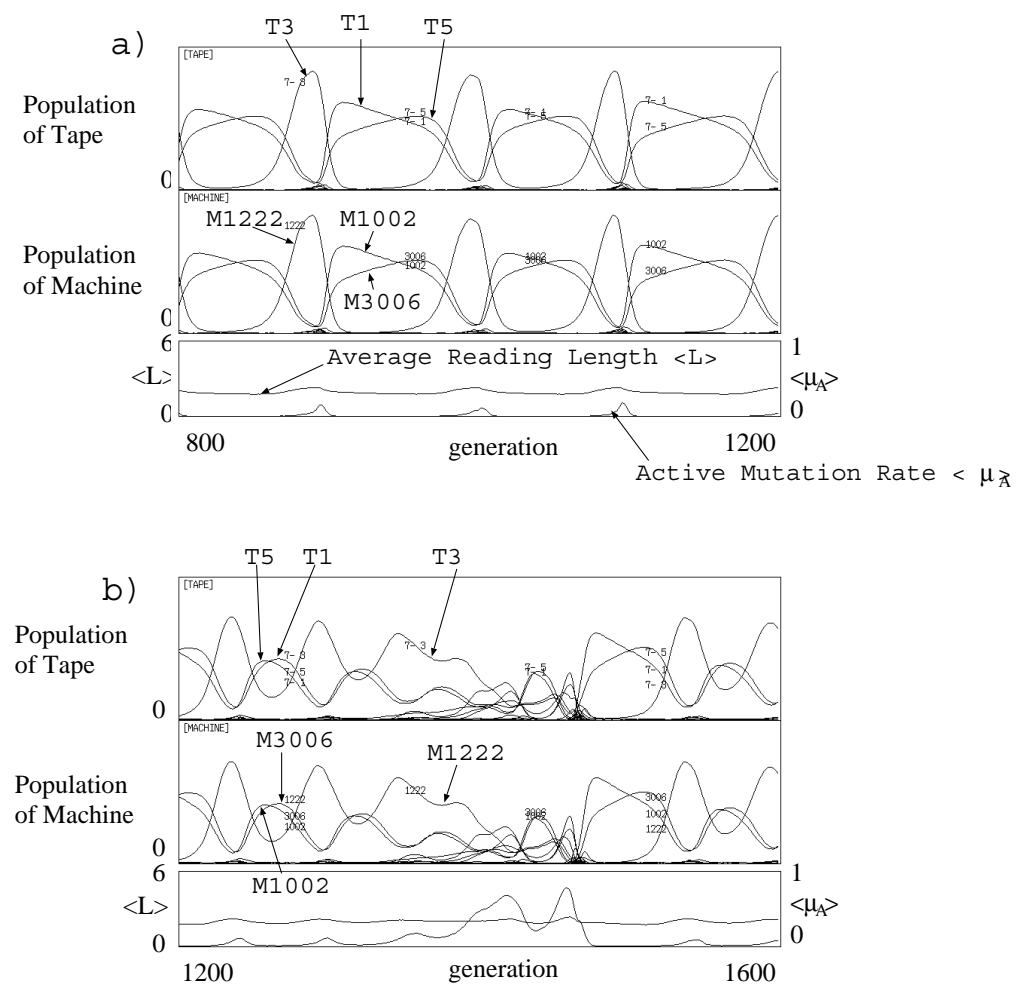

Figure 2: Temporal evolution of population of tapes (the top row) and machines (the middle row). The bottom row displays temporal evolution of averaged active mutation rates and the averaged length of the reading frame. The parameters of population dynamics are $c=d_{m / t}=0.6$ in the influence of, a) lower external noise $\left(\mu_{P}=0.04\right)$ and $\left.\mathrm{b}\right)$ higher external noise $\left(\mu_{P}=0.055\right)$. Both start from the same initial states. 
unstable oscillation in time. Oscillating lines of the larger amplitude are of the original self-replicating loop and a parasite which are depicted in Fig. 2a). Other oscillation lines are caused by parasitic machines and tapes.

These temporal oscillations spontaneously crash by exhausting the original self-replicating loop. The system then restarts if the original selfreplicating loop is retained, otherwise it is extinct.

It should be noted that a non-zero active mutation rate begins to oscillate in time in Fig. 2-b). Lower active mutation mean that the network has more precise local replication; each tape is self-replicated without mutation. But higher active mutation suggest that many reactions produce different tapes from the original ones. Namely, the degree of unsuccessful replication is reflected in the amplitude of active mutation rate.

The initial self-replicating loop shows a zero active mutation rate as being depicted in Fig. 2-a). Intermittent bursts of an active mutation rate are caused by the actions of a parasite machine $\mathbf{M}_{1222}$.

\section{Emergence of Core network}

In the region of middle external noise ( $\left.\mu_{P} \geq 0.05\right)$, a stable structure seems to evolve. Unstable oscillation in population amplitude as we see in Fig. 2-b) is spontaneously stabilized around the generation 600 in Fig. 3. At the same time, the active mutation rate is sustained at the high level.

If we turn off external noise after the generation 600 , the variety of machines and tapes is not completely lost. But if the noise is removed before the generation 600 , it will back to the initial self-replicating loop. We call a network which acquired an implicit stable structure a core network.

Fig. 4 shows temporal evolution of the distinct number of machines and tapes. The amplitude of oscillation becomes less rugged compared with that before a transition. By turning off the noise, the distinct number of machines decreases abruptly to some level. A true core network is left in the system afterwards.

A core network is not necessarily be a fixed point state. It may start to oscillate after turning off the noise. An example of oscillatory state of core network is described in Fig. 5. Both the number of machines and tapes, and an averaged active mutation rate show oscillation after turning off external noise. The distinct number of machines becomes even larger after turning off the noise.

Phenomenologically, core nets as attractors can be divided into roughly 


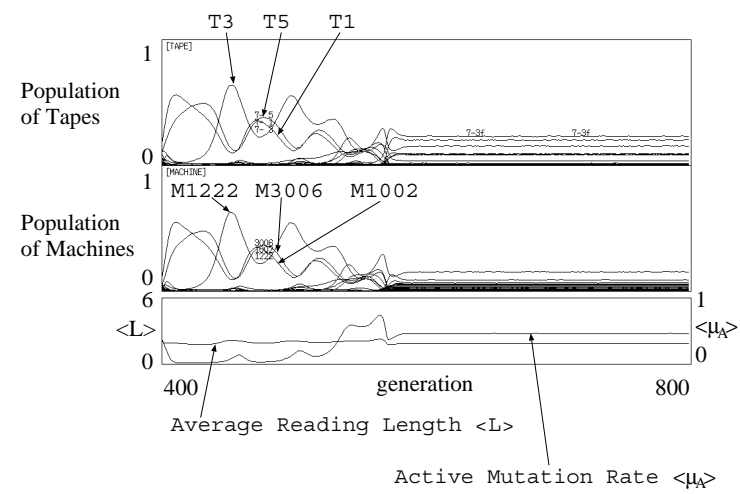

Figure 3: A spontaneous transition into a core network of a fixed point state. It displays temporal evolution of population of tapes (the top row) and machines (the middle row), and averaged active mutation rates and the averaged length of the reading frame (the bottom row). The rate of external noise is set at 0.07 . The parameters of population dynamics are $c=d_{m / t}=0.6$.

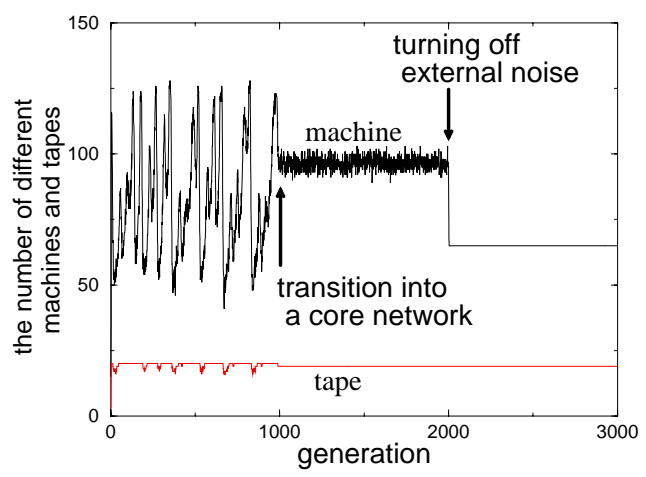

Figure 4: Temporal evolution of distinct numbers of machines and tapes. External noise $\left(\mu_{P}=0.08\right)$ is turned off at the generation 2000 after the emergence of a core network at the generation 1100. The parameters of population dynamics are $c=d_{m / t}=0.6$. 

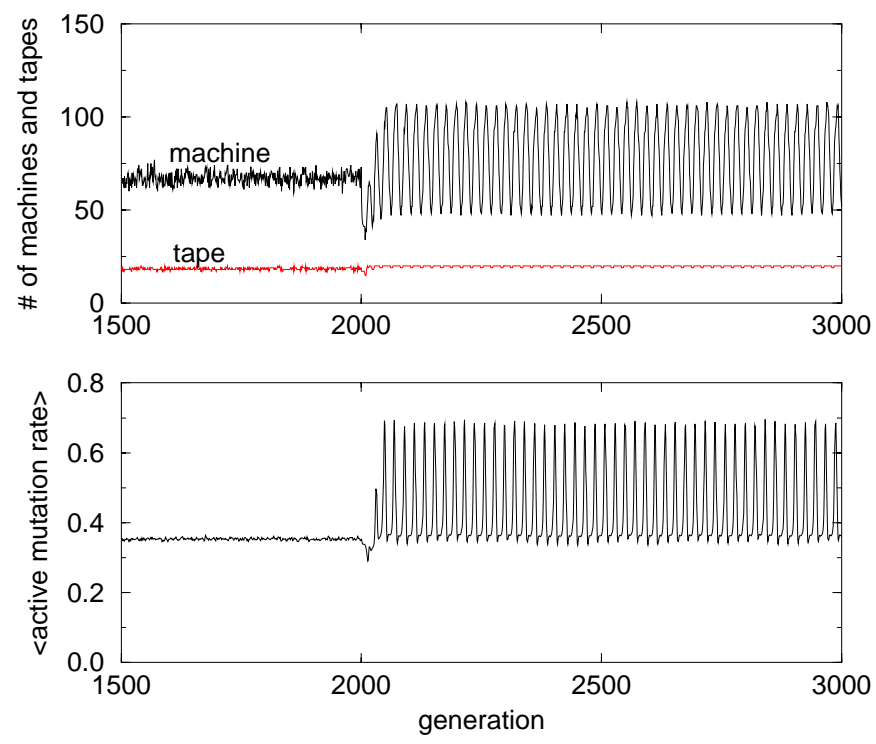

Figure 5: Temporal evolution of distinct numbers of machines and tapes ( top graph) and an averaged active mutation rate (bottom graph). After turning off external noise $\left(\mu_{P}=0.08\right)$ at the generation 2000 , both amounts start to oscillate. The parameters of population dynamics are $c=d_{m / t}=0.6$. 


\section{3 different categories:}

1. Fixed point states A minimal self-replicating loop. Active mutation rate is completely suppressed in this state.

2. Non-trivial fixed point states Population of all machines and tapes in a core network are kept constant. Most core networks have roughly 60 different machines but there is an exceptional core with roughly 40 machines. The constituent machines are very different between two. Active mutation rates are sustained at higher levels.

3. Oscillatory states Population of machines and tapes oscillate in time. Oscillations can be quasi-periodic or almost periodic. Periodicity of the oscillation depends on the set of machines and tapes composing the core networks. Different from the usual notions of periodic behavior, the number of different machines and tapes also change in time. Namely, a topology itself oscillate in time. Here active mutation rates also oscillate in time with keeping a high average value.

There exist upper and lower bound on external noise to evolve rich core networks. Non-trivial cores (i.e. attractor 2 and 3 ) are mostly found at the mid range of external noise $\left(0.05 \leq \mu_{P}<0.1\right)$. In Fig. 6 , we depict active mutation rates of core nets as a function of external noise, which are attained by turning off external noise at the generation 2000. At the higher range of external noise, we again find that a system attains a minimal self-replicating loop (i.e. attractor 1) or being extinct.

This diagram depends on an initial configuration of machines and tapes. Some initial configurations never attain any core networks. Each configuration can be characterized by the existence of self-replicating loops. There exist 5 possible self-replicating loops. We also have studied the evolution from the initial configurations, each of which only contains one of those pairs. It seems that oscillatory states (attractor 3 ) are only attainable when the initial states contains a machine $\mathbf{T}_{\mathbf{9 d d}} \mathbf{3}$ with its own tape $\mathbf{T}_{\mathbf{1 d}}$ or a machine $\mathbf{M}_{\text {bdd1 }}$ with $\mathbf{T}_{\mathbf{3 7}}$.

The initial states which contain each of other three pairs (i.e. $\mathbf{M}_{\mathbf{1 0 0 2}}$ with $\mathbf{T}_{1}, \mathbf{M}_{\mathbf{2 0 0 4}}$ with $\mathbf{T}_{\mathbf{1}}$ and $\mathbf{M}_{\mathbf{d f f b}}$ with $\mathbf{T}_{\mathbf{3 f}}$ ) will mostly lead to nontrivial fixed point states(attractor 2 ).

Fig. 6 is obtained from the initial states which contains none of those pairs. Every attractor can be seen in this diagram. In particular, a core network appeared at external noise of 0.05 contains a rather smaller number 

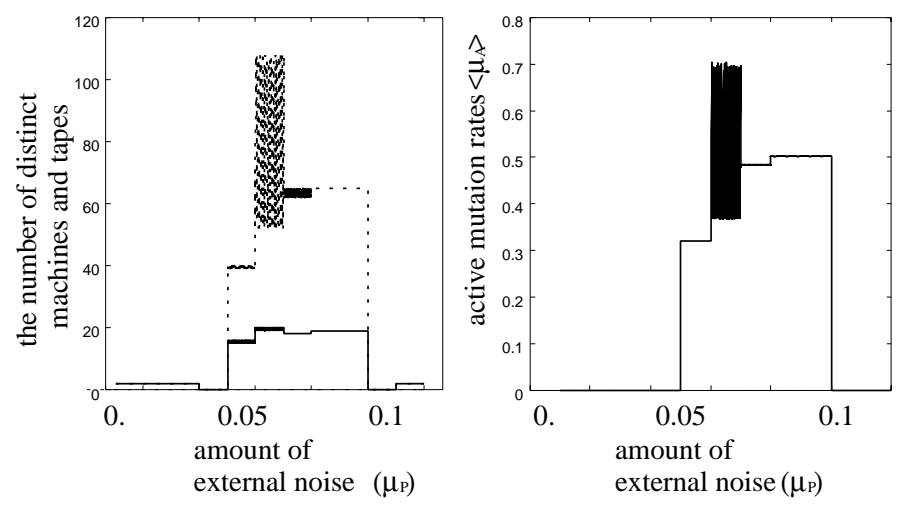

Figure 6: Each column shows final values of the distinct number of machines and tapes (left figure) and those of active mutation rates (right figure) after turning off external noise. Those values are generally oscillating so that we have plotted all points during the generation $[2500,3000]$. A horizontal line corresponds to an amount of external noise added during the generation $[0,2000]$. The noise is turned off at the generation 2000 .

of machines than the usual core networks. We have found that this core uses more 0 -rich tapes, which contains states 0 more than states 1 , in autocatalytic loops. Whereas fixed core networks found in other noise range use more 1-rich tapes. It seems that the amount of a bit of state 1 in tapes used in core networks increases by elevating external noise.

The important criterion to distinguish core networks is to investigate the embedded autocatalytic loops. Since Eigen and Schuster's pioneering work [4], notion of autocatalicity has been known as a useful razor. We use this notion to dissect core networks in the next section.

\section{$5 \quad$ Embedded autocatalytic loops}

A typical evolutionary pathway from the random initial configuration is discussed here. A typical configuration will fall into the configuration which contains a self-replicating pair of a machine $\mathbf{M}_{\mathbf{1 0 0 2}}$ with its own tape $\mathbf{T}_{\mathbf{1}}$. As the most large variety of machines generate the machine $\mathbf{M}_{\mathbf{1 0 0 2}}$ in our our model, the total configuration will often be dominated by this machine.

Evolution from this self-replicating loop to complex ones is depicted in 


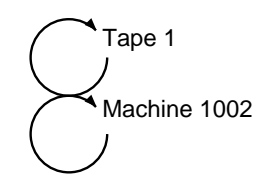

(a)

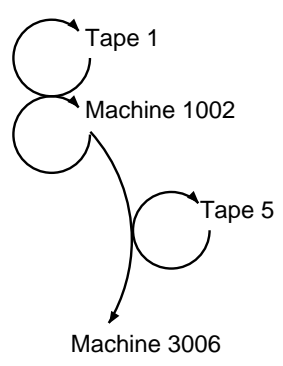

(b)

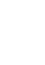<smiles></smiles> 
Fig. 7, where we express it by actual machine-tape reaction graphs. Fig. 7a)c) are corresponding to 2a), and emergence of Fig. 7c) and d) is found in $2 b)$.

In Fig. 7-a), a machine $\mathbf{M}_{\mathbf{1 0 0 2}}$ copies itself by reading its own description tape $\mathbf{T}_{1}$. This self-replicating loop exemplifies Eigen-Schuster's autocatalytic network type. Namely, a tape is replicated into the same tape. In lower noise regime, the minimal loop (a) is gradually destabilized by parasite machines( (a) to (c)). In higher noise regime, parasites as a network appear. Fig. $7 \mathrm{~d}$ ) and e) show a successive appearance of such parasitic networks. We call it a parasite network as it depends its necessary tapes on other networks. As is seen from the figures, a subnet in (d) depends tapes $\left(\mathbf{T}_{\mathbf{3}}\right.$ and $\mathbf{T}_{\mathbf{5}}$ ) on a network (c). Similarly, a subnet on (e) depends a tape $\mathbf{T}_{\mathbf{b}}$ on the parasite network on (d). Hence this latter parasite network can be called a hyper parasite network. Those parasite networks will be extinguished if the original host loop (a) is weaken or removed. Hence the state will be back to (a) and repeat the process from (a) to (e).

Finally, a system acquires a stable structure, which is retained after turning off external noise. A finally established structure is composed of many autocatalytic loops. Fig. 8 shows an example of a core network which is obtained after Fig. 7.

With respect to composing machine sets only, a closed machine loop is identified, where a machine $\mathbf{M}_{j}$ generates a machine $\mathbf{M}_{j+1}$ and the final machine $\mathbf{M}_{k}$ generates the first machine $\mathbf{M}_{1}$. If a necessary tape for each machine is self-produced by one of the machine composing the loop, we say that the loop satisfies an autocatalytic condition.

As special case, the autocatalytic condition is locally satisfied; each tape is self-replicated. A network which satisfies this condition locally is called the Eigen-Schuster type. Since each tape self-replicates, the independent tape loop is identical with the length of a machine loop. However, such autocatalytic condition should not be locally satisfied in general. In the noisy environment, a more general network which globally satisfies the condition can appear.

Difference between two possible types are schematically depicted in Fig. 9. In the case of Fig. 9-b), not only machines but also tapes form a loop structure. Hence we call it a double autocatalytic loop. In the low noise regime, a network can sustain its structure by Eigen-Schuster type. But for the high noise regime, it is difficult to maintain prefect replication. Hence autocatalytic loops switch to the double loop structure. Since Eigen-Schuster type only allows replication without mutation, we call this type a DNA-like repli- 


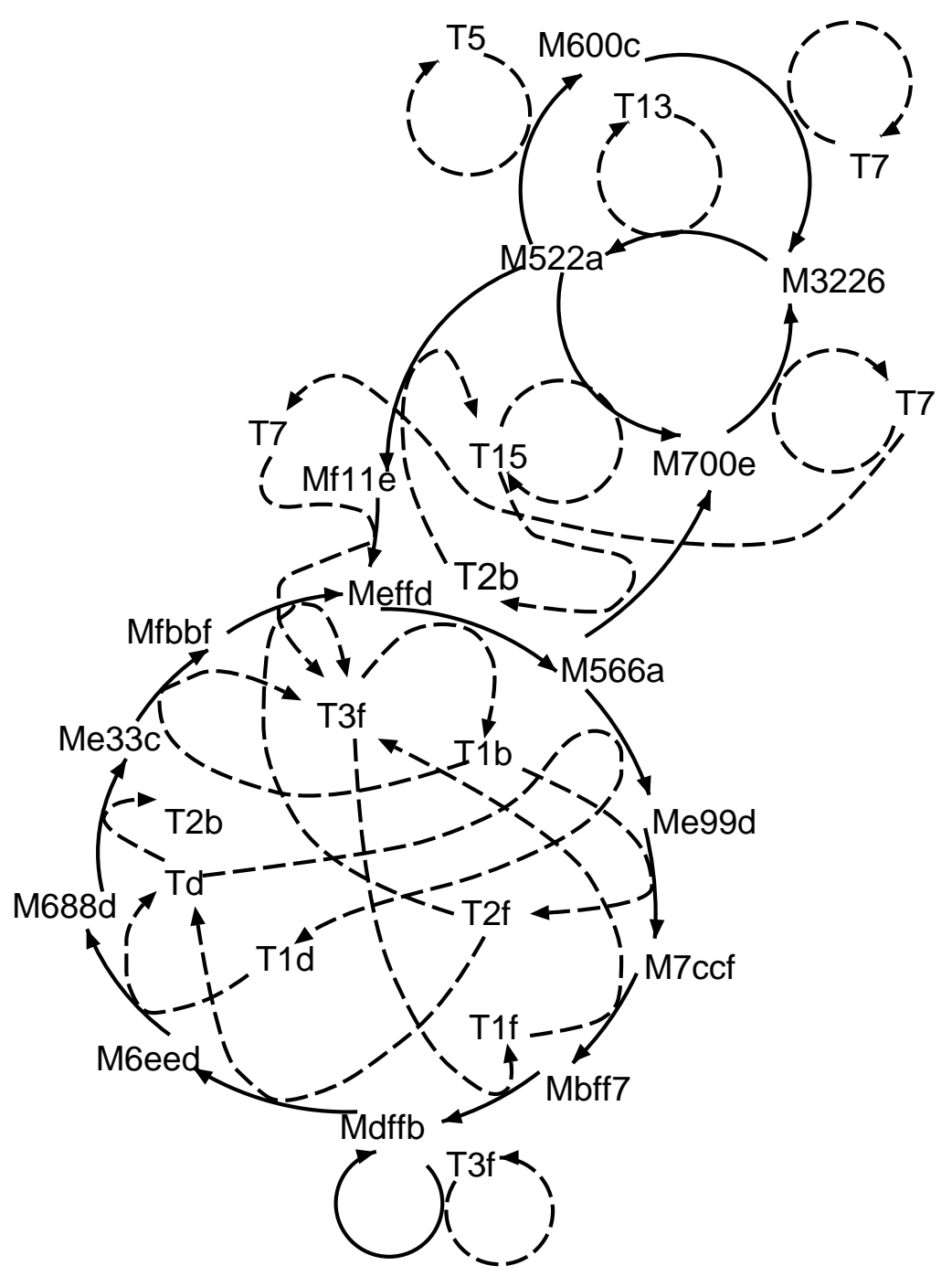

Figure 8: Embedded autocatalytic loops, which are found in the core network after turning off external noise, are depicted. Reducible parasitic subnets are not drawn here. We can see four independent double autocatalytic loops here. Two are of the Eigen-Schuster type and two are of the double loop types. 


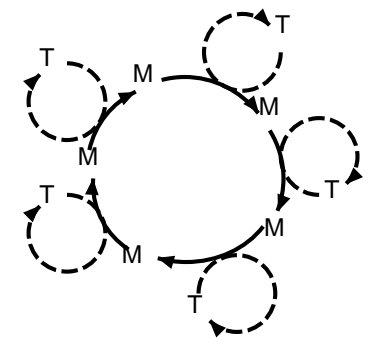

a)

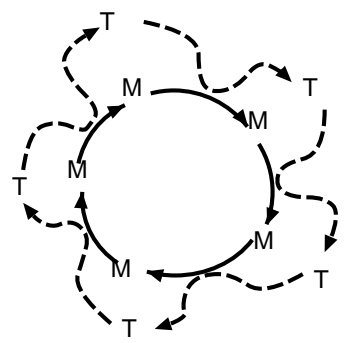

b)

Figure 9: Illustration of two different hyper-cycles. They are Eigen-Schuster type (the right figure) and the double loop type (the left figure). Tapes are replicated without errors in Eigen-Schuster type. In the double loop type, both machines and tapes are respectively forming loops.

cating system. On the other hand, a double loop type edits tapes, which we call a RNA-like editing system. Comparing with core networks in a fixed state, we find that cores in oscillatory states change the number of autocatalytic loops in time. At one period of time a core has only Eigen type but at the different period of time it has doule loop types. Details will be discussed elsewhere [11].

\section{6 discussions}

We have studied the coevolution of machines and their description tapes. We note that a minimal self-replicating loop, composed of one machine and one tape, emerges under influence of external noise.

When external noise is elevated, a minimal self-replicating loop is exploited by parasitic networks. Population of machines and tapes then begins to show unstable oscillations. In the mid regime of external noise, a system will evolve into a stable network against external noise spontaneously. When a network attains this state, it is stably sustained even after external noise is removed. We therefore call the network acquiring such noise stability a "core network". The core network consists of many autocatalytic loops, being a fixed point or oscillatory state. 
A minimal self-replicating loop has no replication error, and corresponding to the simplest Eigen-Schuster's autocatalytic network. Such autocatalytic network is only stable below some noise level. On the other hand, a high active mutation rate is sustained in core networks in the high noise regime. Self-replication with errors is replaced by those with active mutation in the noisy environment.

This autocatalytic network with high active mutation rates consists of double loops of machines and tapes. Namely, each machine replicates a successive machine on a loop. On the other hand, description tapes of the machines composing of a loop are replicated as a whole, not as individual tapes.

A transition from an unstable state to a core network state is similar to what we have seen in a previous host parasite model $[14,15]$. In the model, chaotic instability is shared by almost all species by sustaining a high mutation rate, leading to weak high-dimensional chaos, termed "homeochaos". In this paper, a role of host and parasite emerges spontaneously in a network. Interaction of host and parasite loops causes dynamic instability as well. The core structure suppresses the instability and as the result high mutation rates are sustained in the present model.

The idea of introducing two different mutations provides a new interpretation of self-replication. A network absorbs external noise as active mutation of machine function. Namely, passive mutation caused by external noise is replaced by active mutation.

In real biological systems, DNA is replicated individually without active mutation. RNA is also a mere copy of DNA. However, we have an example [10] which may be related to our double autocatalytic network. The authors showed that DNA of macro-nucleus is generated from DNA of micro-nuclear in Oxytricha nova. DNA of micro-nuclear is transcribed once into RNA. Then the exons are completely rearranged and reverse transcribed into the DNA of macro-nucleus. If editing and reverse transcribing RNA is more stable than replicating DNA itself, active mutation as editing will be favored in some genetic systems.

In order to enhance a desirable mutation, we have to generate mutation not by chance but by a deterministic process. In order to suppress the undesirable mutation, we have to change our coding structure, for example by replacing unstable description tape with more stable tapes. However it is difficult to chose stable coding generally. An optimal coding of a machine may lead to the worst coding of the other. This causes a temporal oscillation even in a core network. Note that self-organization of an autocatalytic 
network implies self-organization of a coding system [20,5]. How to improve coding cannot be determined locally but should be done in a network context. Alternation of coding driven by external noise has also been reported [12]. In their systems, a multi coding system is developed in the high noise regime.

In the light of evolution in formal systems theory, Fontana's Alchemy $[7,8,9]$ shares common features with our system. His level 0 system is corresponding to our simple fixed state core and a level 1 to stable core structures. Instead of meta-inhibition of self-copying in Fontana's model, we have introduced external noise to breed core structures. A level 2 corresponds to inter-cellular interacting system, where each cell contains a full set of core network. If we study interactions among different core networks, what Fontana calls a "glue" sentence is corresponding to machines exchanging between core networks. These glue machines are indeed found in our cell models. Such glue machines are used to make cells of core networks differentiate[13].

\section{Acknowledgment}

One of the authors (T.I.) would like to thank Pauline Hogeweg for stimulating and critical discussions on this work, and her bioinformatic group for their heartful courtesy while he was staying in Utrecht. His stay was supported by NWO (Netherlands Scientic Research Foundation). This work is partially supported by Grant-in-Aids (No.06854014 and 07243102) for Scientific Research from the Japanese Ministry of Education, Science and Culture.

\section{References}

[1] Andrade M, Nuẽo, J., Morán, F., Montero, F. and Mpitos, G. (1993). Complex dynamics of a catalytic network having faulty replication into error-species. Physica D 63, 21-40.

[2] Eigen, M. (1993). Viral quasispecies. Scientific American, July, 32-39.

[3] Schuster, P. (1986). Dynamics of molecular evolution. Physica 22D, $100-119$.

[4] Eigen M. and Schuster P. (1979). Hypercycle. Berlin: Springer-Verlag. 
[5] Eigen, M., Gardiner, W., Schuster, P. and Winkler-Oswatitsch, R. (1981). The origin of genetic information. Scientific American, 244, $78-94$.

[6] See e.g., Farmer D.J., Kauffman S.S., Packard N.H. and Perelson A.S., Ann.Rev. NY Acad. Sci. 504 (1987) 118.

[7] Fonatana W. and Buss L.W., Proc.Natl.Acad.Sci. USA 91 (1994) 757 761.

[8] also see, Bull. Math. Biol. 56(1994) 1-64.;

[9] Fontana, W. (1991). Algorithmic chemistry. In C. G. Langton, C. Taylar, J. D. Farmer and S. Rasmussen (Eds.), Artificial Life II, pp. 159207, Redwood City, CA: Addison-Wesley.

[10] Greslin, A., Prescott D., Oka, Y., Loukin, S. and Chapell, J. (1989). Reordering of nine exons is necessary to form a functional action gene in Oxytricha nova. Proceedings of Natural Academy of Science, USA, $86,6264-6268$.

[11] Hashimoto T. and Ikegami T., to be submitted.

[12] Hogeweg, P. and Hesper, B. (1992). Evolutionary dynamics and the coding structure of sequences: multiple coding as a consequence of crossover and high mutation rates. Computers Chemistry, 16, 171-182.

[13] Ikegami T. and Hashimoto T. (1996) Replication and Diversity in Machine-Tape Coevolutionary Systems. (preprint, accepted to Alife V).

[14] Kaneko, K. and Ikegami, T. (1992). Homeochaos: dynamics stability of a symbiotic network with population dynamics and evolving mutation rate. Physica, 56D, 406-429.

[15] Ikegami, T. and Kaneko, K. (1992). Evolution of host-parasitoid network through homeochaotic dynamics. Chaos, 2, 397-407.

[16] This is a main theme discussed by Hofstadter D. in his book, (1980) Gödel, Escher, Bach: An eternal Golden Braid. New York: Basic Books.

[17] Langton C. (1984). Self-Reproduction in Cellular Automata Physica, 10D, 135. 
[18] Tempesti, G. (1995). A New Slef-Reproducing Cellular Automaton Capable of Construction and Computation. In F. Morán, A. Moreno, J. J. Merelo and P. Chacón (Eds.) Advances in Artificial Life, pp. 555-563 Berlin: Springer.

[19] von Neumann, J. (1968). Theory of Self-reproducing Automata. Urbana: the University of Illinois Press.

[20] Wills, P. (1993). Self-organization of genetic coding. Journal of Theoretical Biology, 162, 267-287. 